\title{
EDITORIAL
}

\section{MEJORAR LA TRANSPARENCIA Y LA CREDIBILIDAD DE LOS ANÁLISIS COSTE-EFECTIVIDAD DE LAS INTERVENCIONES SANITARIAS}

Ferrán Catalá-López $(1,2,3)$ y Manuel Ridao (4,5).

(1) Departamento de Medicina, Universidad de Valencia/Instituto de Investigación Sanitaria INCLIVA y CIBERSAM. Valencia. España

(2) Fundación Instituto de Investigación en Servicios de Salud. Valencia. España

(3) Clinical Epidemiology Program. Ottawa Hospital Research Institute (OHRI). Ottawa. Ontario, Canada.

(4) Instituto Aragonés de Ciencias de la Salud (I+CS). Red de Investigación en Servicios de Salud en Enfermedades Crónicas (REDISSEC). Zaragoza. España

(5) FISABIO-Salud Pública. Valencia. España

La evaluación económica de las intervenciones sanitarias, y más concretamente, su análisis coste-efectividad, se ha posicionado como una herramienta de ayuda en los procesos de toma de decisiones en la asignación de recursos y en la incorporación de tecnologías sanitarias con cargo a fondos públicos ${ }^{1-3}$. El análisis coste-efectividad permite la evaluación comparativa de opciones alternativas atendiendo a los costes y sus consecuencias sanitarias $^{1}$. Revisiones sistemáticas de la calidad de los análisis coste-efectividad publicados en la literatura científica ponen de manifiesto hallazgos, cuanto menos, preocupantes $^{4-7}$. En este sentido, en una reciente revisión sistemática ${ }^{7}$ que examinó una muestra de 223 análisis coste-efectividad realizados en España (publicados hasta diciembre de 2014), en los que la medida de efectividad fueron los años de vida ajustados por calidad (AVAC), se observaron una serie de problemas metodológicos graves. Por mencionar algunos ejemplos, sólo el 5\% de los trabajos indicaron la existencia de un protocolo de investigación, menos de la mitad (47\%) presentaron

\footnotetext{
Correspondencia:

Dr. Ferrán Catalá-López

Departamento de Medicina

Facultad de Medicina y Odontología

Universidad de Valencia

Avda. Blasco Ibáñez no 15

46010 Valencia

ferran_catala@hotmail.com
}

una descripción adecuada de las características de la población estudiada, el 19\% describieron de manera completa los métodos de cálculo para los AVAC y el 18\% presentaron estimadores de efectividad (o eficacia) de las intervenciones utilizando para ello síntesis de la mejor evidencia a partir de revisiones sistemáticas y metaanálisis. Por otro lado, el $40 \%$ de los estudios no presentaron información sobre los conflictos de interés de los autores firmantes de los trabajos. En las situaciones en que por escasa claridad o por ausencia de reporte se omiten aspectos clave de los métodos y resultados de la investigación, efectuar cualquier valoración sobre la validez de las conclusiones del estudio se ve seriamente dificultado ${ }^{8,9}$. Si clínicos, pacientes, investigadores, evaluadores de tecnología sanitaria, planificadores y otras personas interesadas no pueden analizar críticamente la información procedente de los análisis coste-efectividad es difícil que este conocimiento sea de utilidad y que, por tanto, se pueda incorporar a la atención sanitaria y a la toma de decisiones en salud pública.
Cita sugerida: Catalá-López F, Ridao M. Mejorar la transparencia y la credibilidad de los análisis coste-efectividad de las intervenciones sanitarias. Rev Esp Salud Pública. 2016; Vol. 90: 11 de abril d: e1-e4. 
Durante décadas se han llevado a cabo algunas iniciativas para mejorar la claridad y transparencia de la elaboración de artículos científicos $^{10-17}$. Un ejemplo claro son las propuestas de estandarización metodológica o guías de publicación de la investigación ${ }^{18,19}$. Generalmente, estas guías especifican en forma de listas de comprobación o checklists los elementos mínimos necesarios para presentar la información relativa a una investigación de manera clara y coherente. Los ejemplos más conocidos son Consolidated Standards of Reporting Trials CONSORT (CONSORT) para ensayos clínicos ${ }^{10,11}$, STrengthening the Reporting of OBservational studies in Epidemiology (STROBE) para estudios observacionales $^{13,14}$ y Preferred Reporting Items for Systematic Reviews and Meta-Analyses (PRISMA) para revisiones sistemáticas y metaanálisis ${ }^{15}$. Del mismo modo, para la elaboración de publicaciones que presentan análisis costeefectividad existen diversos recursos a nivel nacional $^{20-22}$ e internacional ${ }^{23-28}$, destacando principalmente la declaración CHEERS (Consolidated Health Economic Evaluation Reporting Standards) $)^{27,28}$, que pretende asistir a los autores en la elaboración del artículo, con aspectos que deberían considerarse ya desde el diseño en la planificación de la investigación, y a las personas que los evalúan en su revisión, mejorando así los aspectos relevantes de la publicación de los estudios.

Siguiendo la filosofía y el formato establecido por las normas CONSORT, la declaración CHEERS (accesible a través de la página web de la red EQUATOR: http://www.equatornetwork.org/reporting-guidelines/cheers/) presenta una lista de comprobación con 24 elementos mínimos (o ítems) necesarios para presentar la información relativa a una evaluación económica de manera completa, clara y transparente ${ }^{27}$. Concretamente, CHEERS se centra explícitamente en aspectos de presentación del diseño del estudio, su realización y análisis además de otros que pueden servir para evaluar posibles riesgos de sesgo en los métodos y resultados presentados en el artículo publicado. La publicación en 2013 de la lista de comprobación de la declaración CHEERS fue acompañada de un documento de "explicación y elaboración" 28 en el que se proporcionan algunos ejemplos de publicación adecuada para cada ítem de la lista de comprobación, además de presentar su justificación.

Una manera de mejorar la calidad de la publicación de los análisis coste-efectividad, y su credibilidad ${ }^{29,30}$ es que autores, revisores y editores de revistas científicas sigan, apoyen e implanten eficazmente las guías de publicación, situación que parece ser subóptima en la actualidad. Los comités editoriales de las revistas pueden requerir a los autores que, como parte del proceso de publicación, incluyan una lista de comprobación que indique dónde se presenta cada ítem o punto de la guía. Del mismo modo, los editores también pueden pedir a los revisores que utilicen las guías durante la revisión y evaluación de manuscritos. La adopción, total o parcial, de la declaración CHEERS ya es un requerimiento editorial por parte de prestigiosas revistas biomédicas a través de su incorporación a las normas de publicación (como the BMJ, BMC Medicine, Pharmacoeconomics, International Journal of Technology Assessment in Health Care y Value in Health, entre otras). Disponer o promover el acceso a estas guías de publicación para autores, revisores y editores puede ayudar a corregir algunas de las deficiencias o lagunas de información presentes en la publicación de los análisis coste-efectividad de intervenciones sanitarias.

La promoción del acceso y la utilización de las guías de publicación recomendadas es una prioridad de la política editorial de la Revista Española de Salud Pública en su objetivo de fomentar mejores prácticas de investigación en ciencias de la salud y en salud pública. Las guías de publicación (como la declaración CHEERS) representan una oportunidad que puede servir de ayuda a la comunidad de autores e investigadores que llevan a cabo análisis coste-efectividad en el ámbito del Sistema Nacional de Salud, pero también 
para los revisores y editores de las revistas españolas para que mejoren su adherencia a las guías internacionales de publicación. Mejorar la calidad de la presentación y la credibilidad de la publicación de los análisis coste-efectividad de intervenciones sanitarias requiere la colaboración de todos (autores, revisores $\mathrm{y}$ editores). En última instancia, ello debería contribuir a alcanzar publicaciones de los análisis coste-efectividad completas, claras y transparentes que faciliten la utilización del conocimiento generado y la traslación de la mejor evidencia clínica-económica a los servicios de salud y a la salud pública.

\section{BIBLIOGRAFÍA}

1. Pinto-Prades JL, Ortún-Rubio V, Puig-Junoy J. El análisis coste-efectividad en sanidad. Aten Primaria. 2001;27:275-8.

2. Puig-Junoy J, Peiró S. De la utilidad de los medicamentos al valor terapéutico añadido y a la relación coste-efectividad incremental. Rev Esp Salud Publica. 2009;83:59-70.

3. Catalá-López F, García-Altés A. Evaluación económica de intervenciones sanitarias en España durante el periodo 1983-2008. Rev Esp Salud Publica. 2010;84(4):353-69.

4. Neumann PJ, Stone PW, Chapman RH, Sandberg EA, Bell CM. The quality of reporting in published cost-utility analyses, 1976-1997. Ann Intern Med. 2000;132:964 972.

5. Jefferson T, Demicheli V, Vale L. Quality of systematic reviews of economic evaluations in health care. JAMA. 2002;287:2809-2812.

6. Bell CM, Urbach DR, Ray JG, Bayoumi A, Rosen AB, Greenberg D, et al. Bias in published cost effectiveness studies: systematic review. BMJ. 2006;332:699-703.

7. Catalá-López F, Ridao M, Alonso-Arroyo A, GarcíaAltés A, Cameron C, González-Bermejo D, et al. The quality of reporting methods and results of cost-effectiveness analyses in Spain: a methodological systematic review. Syst Rev. 2016;5:6.

8. Catalá-López F, Peiró S, Hutton B, Pérez Andrés C, Moher D. Declaration of transparency: promoting a more complete, honest and adequate publication of scientific articles. Rev Esp Salud Publica. 2014;88:181-6.

9. Catalá-López F, Hutton B, Moher D. Declaration of transparency for scientific publications. Med Clin (Barc). 2014;142:554-5.
10. Begg C, Cho M, Eastwood S, Horton R, Moher D, Olkin I, et al. Improving the quality of reports on randomized controlled trials. Recommendations of the CONSORT Study Group. Rev Esp Salud Publica. 1998;72:511.

11. Schulz KF, Altman DG, Moher D; CONSORT Group. CONSORT 2010 statement: updated guidelines for reporting parallel group randomised trials. PLoS Med. 2010;7(3):e1000251.

12. Moher D, Cook DJ, Eastwood S, Olkin I, Rennie D, Stroup DF. Improving the quality of reports of metaanalyses of randomized controlled trials: the QUOROM Statement. Rev Esp Salud Publica. 2000;74:107-18.

13. von Elm E, Altman DG, Egger M, Pocock SJ, Gøtzsche PC, Vandenbroucke JP; Iniciativa STROBE. The Strengthening the Reporting of Observational Studies in Epidemiology (STROBE) statement: guidelines for reporting observational studies. Rev Esp Salud Publica. 2008;82:251-9.

14. von Elm E, Altman DG, Egger M, Pocock SJ, Gøtzsche PC, Vandenbroucke JP. STROBE Initiative. The Strengthening the Reporting of Observational Studies in Epidemiology (STROBE) statement: guidelines for reporting observational studies. PLoS Med. 2007;4(10):e296.

15. Moher D, Liberati A, Tetzlaff J, Altman DG; PRISMA Group. Preferred reporting items for systematic reviews and meta-analyses: the PRISMA statement. PLoS Med. 2009;6(7):e1000097.

16. Moher D, Shamseer L, Clarke M, Ghersi D, Liberati A, Petticrew M, et al. Preferred reporting items for systematic review and meta-analysis protocols (PRISMA-P) 2015 statement. Syst Rev. 2015;4:1.

17. Hutton B, Salanti G, Caldwell DM, Chaimani A, Schmid CH, Cameron C, et al. The PRISMA extension statement for reporting of systematic reviews incorporating network meta-analyses of health care interventions: checklist and explanations. Ann Intern Med. 2015;162(11):777-84.

18. Simera I, Altman DG, Moher D, Schulz KF, Hoey J. Guidelines for reporting health research: the EQUATOR network's survey of guideline authors. PLoS Med. 2008;5(6):e139.

19. Altman DG, Moher D. Developing guidelines for reporting healthcare research: scientific rationale and procedures. Med Clin (Barc). 2005;125 Suppl 1:8-13.

20. Rovira J, Antoñanzas F. Economic analysis of health technologies and programmes. A Spanish proposal for methodological standardisation. Pharmacoeconomics. 1995;8:245-52. 
21. Abellán Perpiñán JM, Sánchez Martínez FI, Martínez Pérez JE. La medición de la calidad de los estudios de evaluación económica. Una propuesta de "checklist" para la toma de decisiones. Rev Esp Salud Publica. 2009;83(1):71-84.

22. López Bastida J, Oliva J, Antoñanzas F, García-Altés A, Gisbert R, Mar J, et al. Propuesta de guía para la evaluación económica aplicada a las tecnologías sanitarias. Gac Sanit. 2010;24:154-70.

23. Mason J, Drummond M. Reporting guidelines for economic studies. Health Econ. 1995;4:85-94.

24. Task Force on Principles for Economic Analysis of Health Care Technology Economic analysis of health care technology. A report on principles. Ann Intern Med. 1995;123:61-70.

25. Drummond MF, Jefferson TO. Guidelines for authors and peer reviewers of economic submissions to the BMJ. The BMJ Economic Evaluation Working Party. BMJ. 1996;313:275-283.

26. Siegel JE, Weinstein MC, Russell LB, Gold MR. Recommendations for reporting cost-effectiveness analyses. Panel on cost-effectiveness in health and medicine. JAMA. 1996;276:1339-1341.

27. Husereau D, Drummond M, Petrou S, Carswell C, Moher D, Greenberg D, et al. Consolidated Health Economic Evaluation Reporting Standards (CHEERS) statement. BMJ. 2013;346:f1049.

28. Husereau D, Drummond M, Petrou S, Carswell C, Moher D, Greenberg D, et al. Consolidated Health Economic Evaluation Reporting Standards (CHEERS)--explanation and elaboration: a report of the ISPOR Health Economic Evaluation Publication Guidelines Good Reporting Practices Task Force. Value Health. 2013;16:23150 .

29. Rennie D, Luft HS. Pharmacoeconomic analyses: making them transparent, making them credible. JAMA. 2000;283:2158-2160.

30. Catalá-López F, Alonso-Arroyo A, AleixandreBenavent R, Ridao M, Bolaños M, García-Altés A, et al. Coauthorship and institutional collaborations on costeffectiveness analyses: a systematic network analysis. PLoS One. 2012;7(5):e38012. 